\title{
Analysis of the learning curve for artificial pneumothorax during an endoscopic McKeown-type resection of oesophageal carcinoma
}

\author{
Yanhong Lu, Rongxin Zhang \\ Department of Thoracic Surgery, The First Affiliated Hospital of University of Science and Technology of China (Anhui Provincial Cancer \\ Hospital), Hefei, China \\ Contributions: (I) Conception and design: Y Lu; (II) Administrative support: R Zhang; (III) Provision of study materials or patients: Y Lu; (IV) \\ Collection and assembly of data: Y Lu; (V) Data analysis and interpretation: Y Lu; (VI) Manuscript writing: All authors; (VII) Final approval of \\ manuscript: All authors. \\ Correspondence to: Yanhong Lu, MM. Department of Thoracic Surgery, The First Affiliated Hospital of University of Science and Technology of \\ China (Anhui Provincial Cancer Hospital), Hefei 230000, China. Email: ziyanfengyuan@163.com.
}

\begin{abstract}
Background: Due to the large trauma caused by conventional open surgery, minimally invasive esophageal cancer surgery has been gradually carried out, and there is no report on the learning curve for artificial pneumothorax during an endoscopic McKeown-type resection of oesophageal carcinoma.

Methods: Forty cases of McKeown resection of oesophageal carcinoma with artificial pneumothorax that were completed by the same operator between December 2017 and August 2019 were analysed. The patients were divided into four groups (A, B, C, D) of 10 cases each according to the order of operation. The operation time, intraoperative blood loss, total lymph nodes and left recurrent laryngeal nerve lymph nodes resection, conversion rate, complication rate and hospitalization time were compared between the four groups.

Results: The operation time of the four groups were as follows: A, 243.2 $\pm 44.1 \mathrm{~min}$; B, 265.0 $\pm 59.3 \mathrm{~min}$; C, $255.8 \pm 41.7 \mathrm{~min}$; D, 201.0 $\pm 16.2 \mathrm{~min}$, there were significant difference in terms of the operation time between group A, group B, group C and group $\mathrm{D}(\mathrm{P}<0.05)$. Moreover, groups A and $\mathrm{C}$ all differed significantly from group $\mathrm{D}$ in the number of dissected left recurrent laryngeal nerve lymph nodes. However, no significant inter-group differences were observed in the number of trans-laparotomy and trans-thoracotomy, number of dissected total lymph nodes, intraoperative blood loss, incidence of postoperative complications and postoperative length of hospital stay $(\mathrm{P}>0.05)$.
\end{abstract}

Conclusions: Artificial pneumothorax during an endoscopic McKeown-type resection of oesophageal carcinoma required a learning curve of approximately 30 cases.

Keywords: Learning curve; thoracoscope; laparoscope; artificial pneumothorax

Submitted Dec 15, 2019. Accepted for publication Aug 20, 2020.

doi: $10.21037 /$ tcr-19-2813

View this article at: http://dx.doi.org/10.21037/tcr-19-2813

\section{Introduction}

The applications of endoscopy in the field of thoracic surgery have improved continuously since the early 1990s, and in recent years this technique is widely used in clinical practice. Endoscopic resection of oesophageal carcinoma was reported for the first time in 2000 by Luketich (1). Today, endoscopic resection of oesophageal carcinoma is considered as a safe and feasible procedure that reduce intraoperative blood losses, surgical trauma and the incidence of postoperative complications. Moreover, this procedure is associated with an improved patient quality of life during the early postoperative period and long-term survival rates equivalent in comparison with traditional open surgery.

With the improvement of endoscopic equipment and 


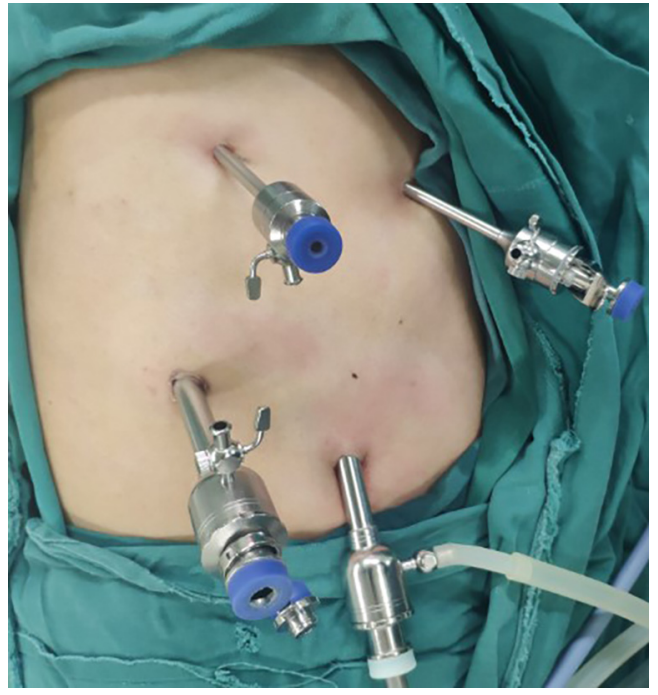

Figure 1 Chest operating hole.

surgical instruments and the optimization of the operative techniques, the scope and depth of endoscopic surgery has been expanded unprecedentedly, which makes the endoscopic McKeown resection procedure for oesophageal carcinoma possible and gradually become the first choice for clinicians and patients. Over time, we observed that the combination of non-artificial pneumothorax and doublelumen endotracheal intubation were associated with a small tissue space, increased bleeding, a longer surgical process and difficulty with exposure of the left laryngeal recurrent nerve chain lymph nodes. Currently, artificial pneumoperitoneum with $\mathrm{CO}_{2}$ is considered safe and effective. Establishment of artificial pneumothorax with $\mathrm{CO}_{2}$ is based on the same principle as that of artificial pneumoperitoneum. During this procedure, $\mathrm{CO}_{2}$ is pumped continuously into the chest cavity at a controlled flow rate, where it enables a stable positive pressure in the pleural cavity that collapses the lung and exposes the operative field.

In December 2017, artificial pneumothorax was commenced applying to thoracoscopic thoracic resection of oesophageal cancer. In this study, we analysed the clinical data of 40 patients who underwent thoracoscopic and laparoscopic oesophageal carcinoma resection via the McKeown procedure with artificial pneumothorax completed by the same operator between December 2017 and August 2019. This study was performed to explore the relevant learning curve associated with this procedure. We present the following article in accordance with the STROBE Reporting Checklist (available at http://dx. doi. org/10. 21037/tcr-19-2813).

\section{Methods}

\section{General information}

Data was collected from 40 cases of patients with oesophageal cancer who underwent thoracoscopy and laparoscopy via the McKeown resection protocol under the condition of artificial pneumothorax at our hospital between December 2017 and August 2019. The cases included 30 men and 10 women with an average age of 66.8 years (range, 42-80 years). All patients underwent preoperative gastroscopy and had pathologically confirmed oesophageal cancer or oesophageal high-grade intraepithelial neoplasia. Moreover, the computed tomography findings suggested that all oesophageal cancers and neoplasias in these cases could be excised with lymph node dissection.

All patients involved in this study gave their informed consent before surgery. The patients were divided into four groups of 10 cases each (A, B, C and D) according to the order of operation. The following clinical data was collected from each group: age, sex, T stage, tumour location and pathological type.

\section{Operation methods}

\section{Chest surgery (Figure 1)}

The patient was placed in the left recumbent position (forward angle: $30^{\circ}$ ) with the upper limb of the affected side raised in front and fixed on a hand bracket. Ideally, the surgeon was located dorsal to the patient. A thoracoscopic hole $(1.0 \mathrm{~cm})$ was made in the midaxillary line in the $7^{\text {th }}$ intercostal region. And then two main operative holes were made: one $(1.0 \mathrm{~cm})$ was located to the posterior axillary line in the $8^{\text {th }}$ intercostal region and the other $(0.5 \mathrm{~cm})$ was located to the posterior axillary line (anterior border of latissimus dorsi) in the $5^{\text {th }}$ intercostal region. Finally, an auxiliary operating hole $(0.5 \mathrm{~cm})$ was made in the $4^{\text {th }}$ intercostal region of the anterior axillary line. Various trocar models were used.

To achieve artificial pneumothorax, the thorax was inflated with $\mathrm{CO}_{2}$ at a low rate $(3 \mathrm{~L} / \mathrm{min})$ to maintain an intrathoracic pressure of $6-8 \mathrm{mmHg}$ and collapse the affected lung. The artificial pneumothorax was maintained throughout the process of thoracic oesophageal and lymph node dissection. A Hem-o-lok device was used to close and cut the azygos vein via thoracoscopy. After electrocautery 


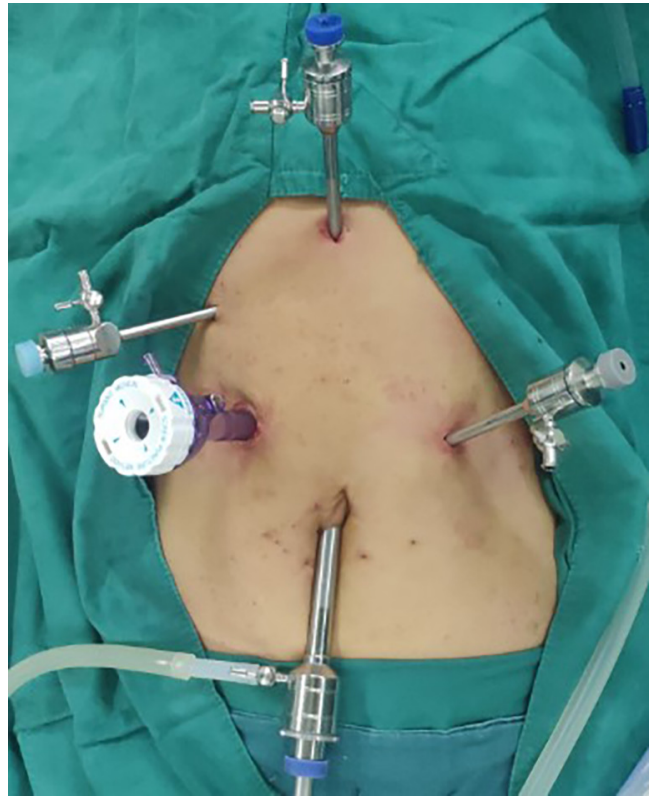

Figure 2 Abdominal operating hole.

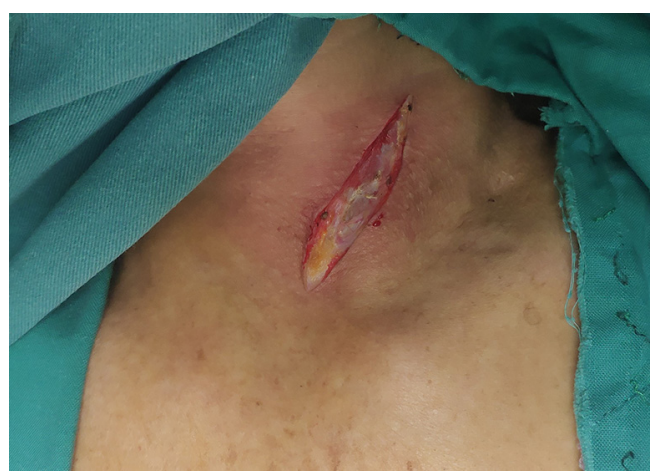

Figure 3 The neck incision.

incision of the mediastinal pleura, dissection of the free thoracic oesophagus and lymph nodes was performed. Oesophageal dissociation, all dissection procedures and azygos vein dissociation and cutting were all completed via the two main operative holes. Following chest dissociation, the chest was closed and a closed thoracic drainage tube was placed in the endoscopic hole.

\footnotetext{
Abdominal operation (Figure 2)

The patient was placed in the supine position with the head elevated and feet placed $30^{\circ}$ downward, as well as under double lung ventilation. $\mathrm{CO}_{2}$ and a pneumoperitoneum needle were used to maintain an intraperitoneal pressure of
}

approximately $15 \mathrm{mmHg}$. A laparoscopic observation hole $(1 \mathrm{~cm})$ was made at the lower margin of the umbilicus. And then, three $5-\mathrm{mm}$ incisions were made as auxiliary operation holes for trocar placement. These were located $2-\mathrm{cm}$ below the xiphoid process, $0.5-\mathrm{cm}$ below the costal margin of the right midline clavicle and $3-\mathrm{cm}$ below the costal margin of the left midline of the clavicle. A 12-mm trocar was inserted into the main operating hole at the midpoint between the right auxiliary operating hole and the laparoscopic observation hole.

The right vascular arch of the gastric omentum was preserved using an ultrasound scalpel. The left gastric artery was severed by using a Hem-o-lok closure via the main operating hole. Gastric dissociation up to the diaphragmatic oesophageal hiatus and down to the pylorus was performed to open the diaphragmatic oesophageal hiatus. The abdominal lymph node was dissected. Next, a 5 -cm incision was made under the xiphoid process. For tumours located in the upper segment, the stomach and oesophagus were isolated from the cardia. For tumours located in the lower segment, the oesophagus was isolated from the neck. The stomach was removed from the abdominal cavity, and a tubular stomach was formed.

\section{Neck operation (Figure 3)}

An incision with an approximate length of $4 \mathrm{~cm}$ was made through the anterior edge of the left sternocleidomastoid muscle. The thoracic oesophagus and tubular stomach were lifted to the left neck after separating the cervical oesophagus. The oesophagus was cut and anastomosed endto-end with the tube stomach.

\section{Background of the performing surgeon}

The surgeon who performed all 40 operations was experienced in the thoracoscopic and laparoscopic resection of oesophageal carcinoma and mastered the operation mode. During the completion of the initial procedures, the surgeon was guided by a mature and experienced surgeon in the field of thoracoscopic and laparoscopic oesophageal carcinoma resection.

\section{Evaluation index}

Inter-group comparisons were made with respect to age, sex, tumour stage, tumour location, operation time, intraoperative blood loss, number of dissected lymph nodes, number of dissected left recurrent laryngeal nerve lymph 
Table 1 General characteristics of 40 cases

\begin{tabular}{|c|c|c|c|c|c|c|c|c|c|c|c|}
\hline Group & $N$ & Age (years) & Gender (M/F) & \multicolumn{5}{|c|}{ T stage } & \multicolumn{3}{|c|}{ Tumor location } \\
\hline$A$ & 10 & $65.3 \pm 10.1$ & $8 / 2$ & 1 & 0 & 2 & 6 & 1 & 1 & 7 & 2 \\
\hline$B$ & 10 & $67.8 \pm 3.9$ & $8 / 2$ & 0 & 4 & 2 & 4 & 0 & 3 & 7 & 0 \\
\hline C & 10 & $70.2 \pm 6.6$ & $9 / 1$ & 1 & 2 & 3 & 4 & 0 & 1 & 2 & 7 \\
\hline $\mathrm{F} / \chi^{2}$ & - & 1.058 & 1.636 & \multicolumn{5}{|c|}{0.756} & \multicolumn{3}{|c|}{1.084} \\
\hline $\mathrm{P}$ & - & 0.379 & 0.198 & \multicolumn{5}{|c|}{0.526} & \multicolumn{3}{|c|}{0.368} \\
\hline
\end{tabular}

Table 2 Perioperative outcomes in the 4 groups

\begin{tabular}{|c|c|c|c|c|c|c|c|}
\hline Group & $\mathrm{n}$ & $\begin{array}{l}\text { Operation } \\
\text { time (min) }\end{array}$ & $\begin{array}{l}\text { Blood loss } \\
(\mathrm{mL})\end{array}$ & $\begin{array}{l}\text { Lymph nodes } \\
\text { (n) }\end{array}$ & $\begin{array}{l}\text { Left recurrent laryngeal nerve } \\
\text { lymph nodes }(n)\end{array}$ & $\begin{array}{c}\text { Postoperative } \\
\text { complication (\%) }\end{array}$ & $\begin{array}{l}\text { Length of } \\
\text { stay (d) }\end{array}$ \\
\hline A & 10 & $243.2 \pm 44.1$ & $195.0 \pm 68.5$ & $25.4 \pm 10.9$ & $0.7 \pm 0.8$ & $1[10]$ & $12.4 \pm 2.0$ \\
\hline C & 10 & $255.8 \pm 41.7$ & $170.0 \pm 48.3$ & $30.6 \pm 7.9$ & $1.1 \pm 1.6$ & $1[10]$ & $11.8 \pm 2.7$ \\
\hline D & 10 & $201.0 \pm 16.2$ & $180 \pm 58.7$ & $30.1 \pm 16.3$ & $3.1 \pm 3.1$ & $2[20]$ & $13.5 \pm 5.0$ \\
\hline$P$ & - & 0.011 & 0.821 & 0.377 & 0.074 & 0.868 & 0.421 \\
\hline
\end{tabular}

nodes, incidence of postoperative complications and length of hospital stay.

\section{Statistical analysis}

Data obtained in this study were all expressed as mean \pm standard error. Results were analyzed by the program SPSS 17.0. Statistical analysis of the experimental data was performed by one-way ANOVA. Differences between two means with $\mathrm{P}<0.05$ was considered statistically significant.

The study was conducted in accordance with the Declaration of Helsinki (as revised in 2013). The study was approved by the Ethics Committee of The First Affiliated Hospital of University of Science and Technology of China (Anhui Provincial Cancer Hospital) (No. 2020-XWK-01), and individual consent for this retrospective analysis was waived.

\section{Results}

\section{Comparison of general data}

There was no significant difference in age, gender, tumor location and tumor invasion depth among the groups $(\mathrm{P}>0.05$, Table 1).

\section{Comparison of operative effects between the four groups (Table 2)}

The difference of operation time among the four groups was statistically significant $(\mathrm{P}<0.05$, Figure 4). Moreover, comparisons of the first two groups $(\mathrm{A}, \mathrm{C})$ with group $\mathrm{D}$ revealed statistically significant differences in the number of dissected left recurrent laryngeal nerve lymph nodes (Figure 5). Surgical complications were observed in six cases, including pulmonary infection in five cases and neck anastomotic fistula in one case. There was no significant difference in the number of dissected total lymph nodes, intraoperative blood loss, incidence of postoperative complications and postoperative length of hospital stay between the groups $(\mathrm{P}>0.05$, Table 2$)$.

\section{Discussion}

Oesophageal cancer is one of the most common 


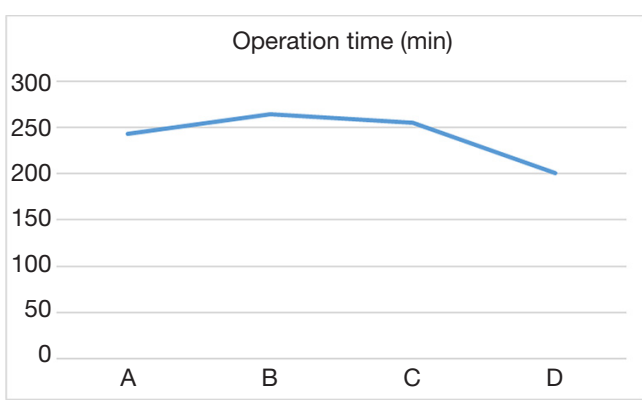

Figure 4 The curve of operation time.

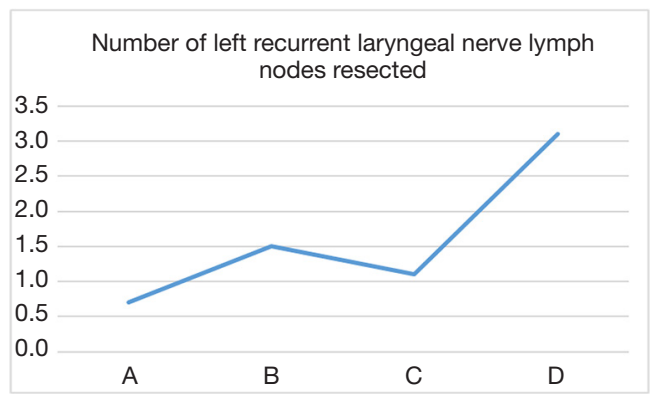

Figure 5 The curve of number of left recurrent laryngeal nerve lymph nodes resected.

malignancies reported in China. The resection of oesophageal cancer involves a large amount of surgical trauma because the procedure requires thoracotomy, digestive tract reconstruction and extensive lymph node dissection. Consequently, this procedure is associated with high rates of postoperative complications and mortality. A minimally invasive and individualised surgical procedure is needed to reduce the incidence of these negative outcomes associated with traditional open surgery.

In China, middle oesophageal carcinoma is more common than lower or upper oesophageal carcinoma. Therefore, the McKeown technique is widely used in China to enable better lymph node dissection and esophagectomy. In a retrospective analysis of 82 patients who underwent a minimally invasive McKeown resection of oesophageal carcinoma, Zhu et al. (2) concluded that this procedure was feasible and safe. However, thoracoscopic surgery is complicated fundamentally by the need for exposure, which improves safety and efficacy. As noted in the introduction, we observed several challenges associated with endoscopic surgery under the condition of non-artificial pneumothorax and double-lumen endotracheal intubation. With artificial pneumothorax (3), however, we could achieve a more rapid and complete lung collapse and increased adipose tissue space in the mediastinum and around the lymph nodes, these improvements facilitated free exposure while reducing blood losses, the operative time was shortened, the postoperative hospital stay was shortened, and more recurrent laryngeal lymph nodes were dissected.

Endoscopic surgery differs from traditional open surgery because rather than directly viewing the operating field, an endoscopic surgeon must rely on a television screen and endoscopic instruments during the procedure. Therefore, the initial implementation of a combined thoracoscopic and laparoscopic surgical procedure requires both initial learning and repeated practice to achieve proficiency. The surgical effect and complication rate improve significantly after a certain number of practical operations, and a relatively stable state is achieved thereafter. The learning stage for an endoscopic procedure is considered the initial stage of surgery, and is usually measured by the number of surgical cases required for mastery during this stage (4). Consequently, the learning curve for an endoscopic surgical procedure is inferred mainly according to the operation time, intraoperative blood loss, rate of conversion to thoracotomy (abdomen), postoperative complications and postoperative length of hospital stay (5). These learning curves differ with respect to surgical position, difficulty and operation style, and may also be affected by the surgical concept, procedural design and understanding of minimally invasive surgical skills. Currently, studies of the learning curves associated with endoscopic surgery have focused mainly on various laparoscopic surgeries. Jung et al. (5) reported that the learning curve for laparoscopic total gastrectomy required approximately 100 cases. In contrast, Binet et al. (6) reported that the learning curve for laparoscopic pyloromyotomy required approximately 10 cases. However, there is still lack of information for the learning curve for artificial pneumothorax during a thoracoscopic and laparoscopic McKeown resection of oesophageal carcinoma.

This study conducted a statistical comparison of 40 consecutive completed cases involving artificial pneumothorax during endoscopic McKeown radical resection of oesophageal cancer. An analysis of the average operation time in each group revealed an obvious downward trend between groups B and D, as well as a statistically significant inflection point in the learning curve between groups $\mathrm{C}$ and $\mathrm{D}$. The operation time fluctuated the most in group A and was shorter than in group B, this observation was attributed to the novelty of the operation technique and 
immaturity of the technology. For example, left recurrent laryngeal nerve lymph node dissection was not performed in the first four cases. After 30 cases, we observed a statistically significant decrease in the average operation time per group. Regarding lymphadenectomy of the left recurrent laryngeal nerve, we observed a statistically significant inflection point in the learning curve between groups $\mathrm{C}$ and $\mathrm{D}$. The results indicate that after 30 surgical procedures, the operation time decreased significantly and the number of dissected left recurrent laryngeal nerve lymph nodes increased significantly. These findings suggest that the learning curve for a radical McKeown resection of oesophageal carcinoma with artificial pneumothorax is approximately 30 cases.

Regarding lymph node dissection, we observed no statistically significant inter-group differences in the numbers of dissected total lymph nodes. However, the number of dissected lymph nodes increased gradually as the level of surgical experience increased. This pattern also suggests that the combination of thoracoscopy with a laparoscopic radical resection of oesophageal cancer is technically feasible.

Every surgeon aims to improve the surgical effect, reduce complications and reduce the number of cases required to achieve the learning curve as soon as possible. The author recommends directing attention to the following aspects during the early stage of the learning process of thoracolaparoscopy combined with radical resection of oesophageal cancer.

(I) Proficiency with thoracoscopy technology and rich theoretical knowledge. Because human aerial thoracoscope oesophagus carcinoma techniques from the complexity of the operation. First, the operator must undergo pre-training before basic training with endoscopy. This includes simulator training for minimally invasive procedures, mediastinal tumours, lung wedge resection of the tumour and other procedures needed to achieve 3-dimensional to 2-dimensional transformation, adapt to the use of a cavity mirror for surgery and location and achieve satisfactory hand-eye coordination. Second, the operator's mastery of surgical theoretical knowledge, the ability to perform and comprehend the operation and aspects of learning and peer-to-peer communication will also affect the learning curve.

(II) Adequate preoperative preparation and selection of surgical patients. Endoscopic oesophageal surgery is associated with relatively long operation times and is considered potentially risky. Therefore, the surgeon must be prepared to address various possible risks and must remain psychologically capable of completing the operation successfully. At the beginning of the learning curve, the surgical indications should be controlled strictly. To the extent possible, the selected patients should have a thin physique, a small tumour with no obvious external invasion or adhesions in chest and abdominal cavity, no obviously enlarged lymph nodes and a good cardiopulmonary condition. These important criteria will help to increase both the learning curve and the surgeon's confidence.

(III) A relatively fixed surgical team and use of appropriate minimally invasive surgical instruments. The rapid developments in endoscopic surgery have been attributed to continuous improvements in the available minimally invasive surgical instruments and equipment and a high level of tacit cooperation within fixed surgical teams.

(IV) Video guided surgery with mature mode, and physicians with a high level of experience with the thoracic laparoscopic resection of oesophageal cancer can provide assistance from the side-lines and enable newer surgeons to achieve a steady state as soon as possible. A mature mode of surgery video can help to achieve the following aims: (i) an understanding of image features and familiarity with anatomical structures; (ii) familiarity with surgical procedures and (iii) the formation of conditioned reflexes, know the attention in the operation.

In conclusion, this study has described the experience of a single surgeon to illustrate the learning curve required for artificial pneumothorax during a thoracic laparoscopic McKeown resection of oesophageal cancer. Certainly, the learning curves will differ among surgeons. For example, Tapias (7) reported a minimum learning curve of 35-40 cases to reach a plateau for minimally invasive esophagectomy. Differences among surgeons may be related to many factors, including surgical methods, tumour stages, previous experience with thoracic laparoscopic surgery and learning abilities.

Endoscopic esophageal surgery is the trend of The Times, and the addition of artificial pneumothorax on the basis of endoscopic surgery is conducive to endotracheal intubation, intraoperative management and better oxygenation (8). Of course, it also has certain disadvantages, 
such as affecting the coagulation function and increasing the risk of tumor metastasis with the spread of $\mathrm{CO}_{2}(8,9)$. There are still many difficulties to be overcome step by step, and we need to continue to work hard.

\section{Acknowledgments}

Funding: None.

\section{Footnote}

Reporting Checklist: The authors have completed the STROBE reporting checklist. Available at http://dx.doi. org/10.21037/tcr-19-2813

Data Sharing Statement: Available at http://dx. doi. org/10. 21037/tcr-19-2813

Conflicts of Interest: Both authors have completed the ICMJE uniform disclosure form (available at http://dx. doi. org/10. 21037/tcr-19-2813). The authors have no conflicts of interest to declare.

Ethical Statement: The authors are accountable for all aspects of the work in ensuring that questions related to the accuracy or integrity of any part of the work are appropriately investigated and resolved. The study was conducted in accordance with the Declaration of Helsinki (as revised in 2013). The study was approved by the Ethics Committee of The First Affiliated Hospital of University of Science and Technology of China (Anhui Provincial Cancer Hospital) (No. 2020-XWK-01), and individual consent for this retrospective analysis was waived.

Open Access Statement: This is an Open Access article distributed in accordance with the Creative Commons Attribution-NonCommercial-NoDerivs 4.0 International License (CC BY-NC-ND 4.0), which permits the non- commercial replication and distribution of the article with the strict proviso that no changes or edits are made and the original work is properly cited (including links to both the formal publication through the relevant DOI and the license). See: https://creativecommons.org/licenses/by-nc-nd/4.0/.

\section{References}

1. Luketich JD, Schauer PR, Christie NA, et al. Minimally invasive esophagectomy. Ann Thorac Surg 2000;70:906-11.

2. Zhu ZY, Yong X, Luo RJ, et al. Clinical analysis of minimally invasive McKeown esophagectomy in a single center by a single medical group. J Zhejiang Univ Sci B 2018;19:718-25.

3. Lin $M$, Shen $Y$, Wang $H$, et al. A comparison between two lung ventilation with $\mathrm{CO} 2$ artificial pneumothorax and one lung ventilation during thoracic phase of minimally invasive esophagectomy. J Thorac Dis 2018;10:1912-8.

4. Tsou YK, Chuang WY, Liu CY, et al. Learning curve for endoscopic submucosal dissection of esophageal neoplasms. Dis Esophagus 2016;29:544-50.

5. Jung DH, Son SY, Park YS, et al. The learning curve associated with laparoscopic total gastrectomy. Gastric Cancer 2016;19:264-72.

6. Binet A, Bastard F, Meignan P, et al. Laparoscopic Pyloromyotomy: A Study of the Learning Curve. Eur J Pediatr Surg 2018;28:238-42.

7. Tapias LF, Morse CR. Minimally invasive Ivor Lewis esophagectomy: description of a learning curve. J Am Coll Surg 2014;218:1130-40.

8. Lin M, Shen Y, Feng M, et al. Is two lung ventilation with artificial pneumothorax a better choice than one lung ventilation in minimally invasive esophagectomy? J Thorac Dis 2019;11:S707-12.

9. Mao QX, Guo W, Huang BQ, et al. Impact of artificial capnothorax on coagulation in patients during videoassisted thoracoscopic esophagectomy for squamous cell carcinoma. Surg Endosc 2016;30:2766-72.
Cite this article as: Lu Y, Zhang R. Analysis of the learning curve for artificial pneumothorax during an endoscopic McKeown-type resection of oesophageal carcinoma. Transl Cancer Res 2020;9(10):5949-5955. doi: 10.21037/tcr-19-2813 